\title{
Differential effects of reticulophagy and mitophagy on nonalcoholic fatty liver disease
}

\author{
Lijun Pang ${ }^{1,2}$, Kai Liu ${ }^{1,2}$, Daojie Liu ${ }^{3}$, Fudong Lv², Yunjin Zang ${ }^{4}$, Fang Xie ${ }^{1,2}$, Jiming Yin $\mathbb{B}^{1,2}$, Ying Shi ${ }^{1,2}$, \\ Yanjun Wang ${ }^{1,2}$ and Dexi Chen ${ }^{1,2,4}$
}

\begin{abstract}
Autophagy affects the pathological progression of non-alcoholic fatty liver disease (NAFLD); however, the precise role of autophagy in NAFLD remains unclear. In this study, we want to identify the role of autophagy including reticulophagy and mitophagy in NAFLD pathogenesis. When HepG2 cells were treated with $400 \mu \mathrm{M}$ oleic acid (OA), increased reticulophagy was induced $8 \mathrm{~h}$ after treatment, which correlated with an anti-apoptotic response as shown by the activation of the PI3K/AKT pathway, an increase in BCL-2 expression, and the downregulation of OA-induced lipotoxicity. When treated with OA for $24 \mathrm{~h}$, DRAM expression-dependent mitophagy resulted in increased apoptosis in HepG2 cells. Inhibition of reticulophagy aggravated and increased lipotoxicity-induced apoptosis $8 \mathrm{~h}$ after treatment; however, the inhibition of mitophagy decreased hepatocyte apoptosis after $24 \mathrm{~h}$ of OA treatment. Results from the analysis of patient liver samples showed that autophagic flux increased in patients with mild or severe NAFL. PI3K/AKT phosphorylation was observed only in samples from patients with low-grade steatosis, whereas DRAM expression was increased in samples from patients with high-grade steatosis. Together, our results demonstrate that reticulophagy and mitophagy are independent, sequential events that influence NAFLD progression, which opens new avenues for investigating new therapeutics in NAFLD.
\end{abstract}

\section{Introduction}

Non-alcoholic fatty liver disease (NAFLD) is a very common public health problem that affects approximately one-third of adults and an increasing number of children in developed countries ${ }^{1}$. NAFLD is most likely promoted by the deregulation of hepatic lipid metabolism as a hepatic manifestation of metabolic syndrome, which clusters visceral obesity, dyslipidemia, hypertension, atherosclerosis, and insulin resistance ${ }^{2}$. NAFLD can progress from simple steatosis (i.e., non-alcoholic fatty liver, NAFL) to non-alcoholic steatohepatitis (NASH), liver

Correspondence: Dexi Chen (dexichen@ccmu.edu.cn)

${ }^{1}$ Beijing Institute of Hepatology, Capital Medical University, 100069 Beijing, China

${ }^{2}$ Beijing You'an Hospital, Capital Medical University, 100069 Beijing, China Full list of author information is available at the end of the article

Lijun Pang and Kai Liu contributed equally to this work.

Edited by G.M. Fimia fibrosis, cirrhosis, and even hepatocellular carcinoma ${ }^{3}$. The exact mechanisms of the NAFL-to-NASH transition remains elusive; however, this transition is a crucial point for NAFL due to the increase in morbidity and mortality upon the development of $\mathrm{NASH}^{4}$. The most prevalent mechanism used to explain the development of NASH is the "multiple hit" hypothesis ${ }^{5}$. According to the multiple hit hypothesis, various hepatic insults result in lipid deregulation and steatosis, as the accumulation of lipid is sufficient to induce lipid peroxidation and inflammation associated with the NAFL-to-NASH transition ${ }^{6}$.

Autophagy-dependent degradation of compromised organelles is a cellular pathway crucial for maintaining cell homeostasis, and organellar autophagy can be selective for the degradation of mitochondria (mitophagy), endoplasmic reticulum (reticulophagy), and lipid droplets 
(lipophagy) $^{7-9}$. Lipophagy is currently regarded as an alternative pathway for lipid metabolism in hepatocytes, and the impairment of lipophagy contributes to NAFLD development ${ }^{7}$. Previous studies have shown that free fatty acid (FFA)-induced lipotoxicity impairs the function of the endoplasmic reticulum (ER) by increasing protein misfolding and by inducing ER stress, which can exacerbate NAFLD $^{10}$. Furthermore, autophagy can lead to cell death and has been shown to be associated with caspase- 3 activation-dependent apoptosis ${ }^{10,11}$. However, the exact mechanisms by which mitophagy, reticulophagy, and lipophagy influence NAFLD progression remain unknown.

Activation of the PI3K/AKT signaling pathway is involved in protecting cells from apoptosis, whereas the activation of the p53 signaling pathway promotes apoptosis $^{12}$. However, both pathways affect the activation of autophagy and have been reported to be associated with NAFLD development ${ }^{13,14}$. PI3K/AKT pathway activation is thought to regulate autophagy through mTOR, and the PI3K inhibitor LY294002 is a general inhibitor for autophagic sequestration ${ }^{13,15}$. However, class III PI3K (VPS34) appears to be involved in autophagosome biogenesis $^{13}$. Following genotoxic stress, p53 becomes activated and inhibits cell proliferation in an effort to maintain genomic integrity ${ }^{16}$. p 53 functions primarily as a transcription factor and induces the expression of several p53 target genes such as p21 and PUMA (p53 upregulated modulator of apoptosis). p21 inhibits several cyclin-dependent kinases, which results in either G1 arrest or G2/M checkpoint arrest. PUMA is a proapoptotic BH3-only BCL-2 family protein that promotes BCL2-associated $\mathrm{X}$ protein (BAX) expression and mitochondria-dependent apoptosis ${ }^{17}$. A relationship between the p53 signaling pathway and autophagy activation was established due in part to the discovery of the p53-dependent regulation of DRAM (damage-regulated autophagy modulator) and Sestrin2, which were subsequently shown to modulate autophagy and promote apoptosis $^{18-20}$. However, basal levels of cytoplasmresident p53 also appear to directly inhibit autophagy ${ }^{21}$.

Considering that lipophagy can regulate lipid metabolism, we hypothesized that autophagy might also play a role in promoting resistance to damage caused by high levels of FFA. However, we failed to identify lipophagy as a contributing factor in the reduction of lipid droplet formation in HepG2 cells subjected to $400 \mu \mathrm{M}$ oleic acid (OA) treatment. In contrast, OA treatment primarily induced reticulophagy, which prevented hepatocytes from undergoing lipotoxicity-induced apoptosis apparently through a decrease in lipotoxicity, the activation of the PI3K/AKT pathway, and an increase in BCL-2 expression. After reticulophagy subsided, OA treatment induced mitophagy, which was mediated by DRAM and played a pro-apoptotic role resulting in hepatocyte death.
Furthermore, we showed that autophagy could be identified in liver samples from patients with low- and highgrade steatosis. However, PI3K/AKT activation could only be identified in liver tissues from patients with low-grade steatosis, whereas DRAM expression could only be identified in liver tissues from patients with high-grade steatosis. The results of our clinical specimen analysis support our in vitro data, which suggest that reticulophagy and mitophagy may be independent, sequential events responsible for the pathological progression of NAFLD, which can trigger both anti- and pro-apoptotic outcomes in hepatocytes.

\section{Results}

Two waves of autophagy can be induced in HepG2 cells at different stages of NAFLD in response to OA stimulus

Autophagy plays a pivotal role in regulating the pathological progression of NAFLD ${ }^{22}$; however, the mechanism remains incompletely understood. Therefore, we sought to identify the role of FFA-induced autophagy on NAFLD development using an in vitro model. Previous studies have shown that OA induces NASH development in HepG2 cells, which is characterized by the accumulation of lipid droplets and the induction of apoptosis at concentrations ranging from 0.1 to $2 \mathrm{mM}^{23-25}$. We treated HepG2 cells with $400 \mu \mathrm{M}$ OA for $24 \mathrm{~h}$ to observe the progression of NAFLD in vitro. The induction of lipid droplet formation was confirmed by Oil Red O staining and by measuring the concentration of intracellular triglycerides (Fig. 1a, b). Moreover, OA significantly increased cell death as determined by the fact that LDH (Lactate Dehydrogenase) release was observed $24 \mathrm{~h}$ posttreatment (Fig. 2b). These results suggest that $400 \mu \mathrm{M}$ OA induces the progression of NAFLD during the 24-h treatment period.

We examined whether autophagy might be associated with the development of NAFLD in HepG2 cells treated with OA. We observed that autophagosome formation reached a peak at 8 and $24 \mathrm{~h}$ post-OA treatment (Fig. 1a), and transfection of HepG2 cells with an empty vector showed no significant change (data not shown). Western blotting results also showed LC3-II level was mainly induced upon 8 and $24 \mathrm{~h}$ of OA treatment (Fig. 1b). The levels of p62 are contrary to LC3-II, which appear to correlate well with other parameters of autophagic flux ${ }^{26}$. In addition, we examined autophagic flux by using BafilomycinA1 (BafA1), which is used to prevent the fusion of autophagosomes with lysosomes. The two waves of autophagy flux OA-induced were blocked by BafA1 obviously. We also obtained similar results in other two types of hepatocytes including 7702 and SMMC-7721 cells (Supplementary Figure 1). Our results showed that the mRNA levels of beclin-1, Atg5, and Atg7 had two peaks except p62 (Fig. 1d). These results suggest that two 


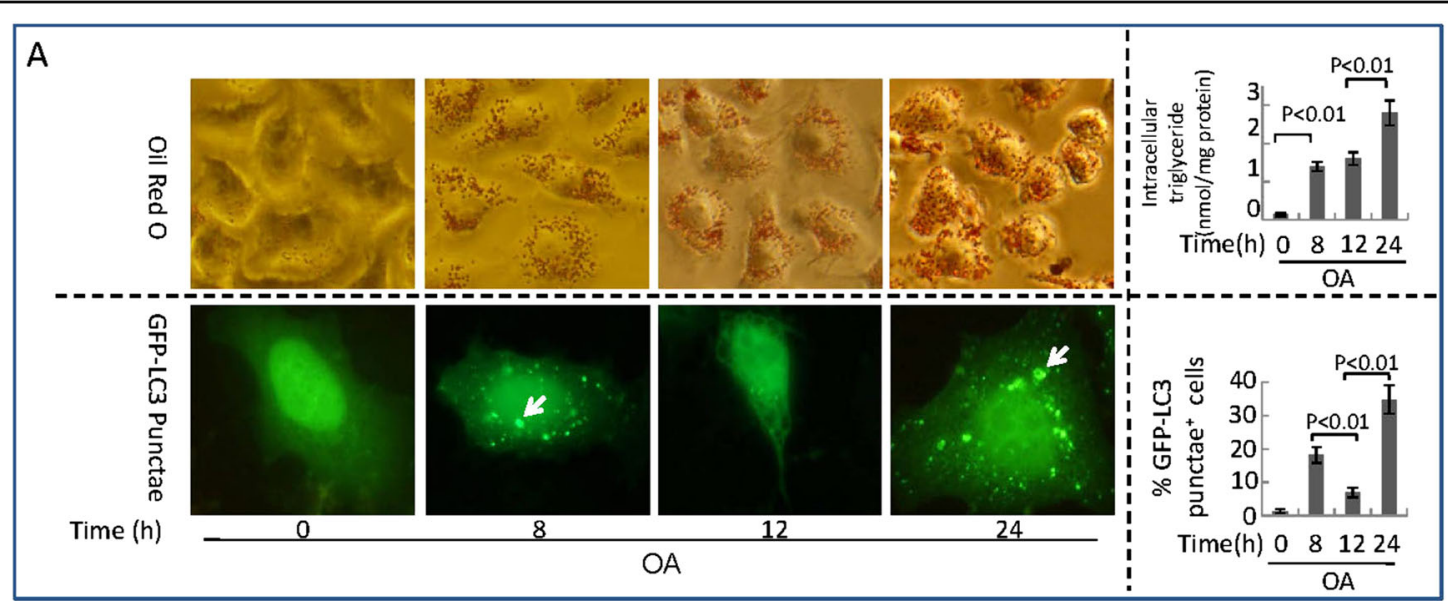

B

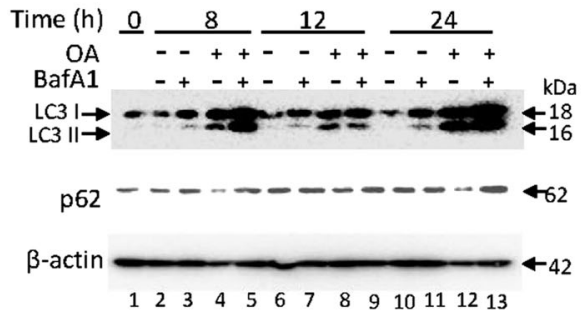

C

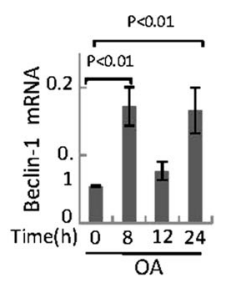

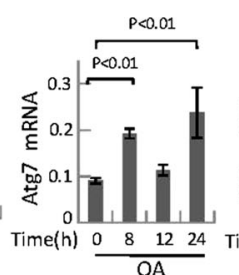

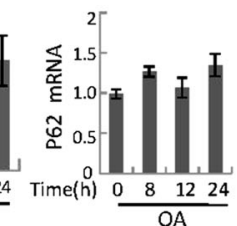

Fig. 1 Autophagy development peaks at 8 and $24 \mathrm{~h}$ in response to $\mathbf{4 0 0} \mu \mathrm{M}$ OA stimulus in HepG2 cells. a Oil Red O staining (upper left panel, original magnification, $\times 400$ ) and intracellular triglyceride levels of cells (upper right panel); representative GFP-LC3 images (lower left panel, original magnification, $\times 1000$ ) and the percentage of autophagosomes (lower right panel) of the cells. White arrow indicates autophagosomes formation and cells with 5 or more GFP-LC3 puncta were considered to have accumulated autophagosomes. Data are presented as mean \pm SEM in three independent experiments. b Representative western blotting analysis of LC3I/II and p62 expressions after using Bafilomycin A1 in cells. c Real-time PCR analysis of mRNA levels of Beclin-1, Atg5 a, Atg7, and p62 in cells. Data are presented as mean \pm SEM in three independent experiments

waves of autophagy are present in the development of NAFLD. Therefore, we sought to identify the role and mechanism of these two waves of autophagic flux.

The first wave of autophagy is mainly reticulophagy and plays a role in protecting hepatocytes from apoptosis; the second wave of autophagy is mainly mitophagy and contributes to hepatocytic apoptosis

Because apoptosis contributes to the pathological progression of NAFLD, we investigated possible connections between autophagy and apoptosis in OA-treated HepG2 cells. We found that OA treatment induced the cleavage of PARP (poly ADP-ribose polymerase) upon 12 and $24 \mathrm{~h}$ of treatment (Fig. 2a). PARP is cleaved by caspase-3 and caspase-7 to yield p85 and p25 PARP fragments, respectively, and the cleavage of PARP has been widely used as an apoptotic marker ${ }^{27}$. Furthermore, we employed calcein AM/PI and M30 immunoreactivity to assess apoptosis. Calcein AM stains for viable cells, whereas PI stains for late apoptotic cells. M30 stains caspase-3-cleaved keratin 18 and thus identifies apoptotic cells. Recently, increased levels of M30 have also been considered to be a marker of
NAFLD development ${ }^{28-30}$. However, HepG2 cell apoptosis was observed $12 \mathrm{~h}$ and reached a peak $24 \mathrm{~h}$ post-OA treatment (Fig. 2c, d and Supplementary Figure 2).

Stress can induce both reticulophagy and mitophagy in cells $^{9,31}$. The ER senses the accumulation of misfolded proteins caused by lipotoxicity and can induce reticulophagy $^{31}$. Although mitophagy maintains intracellular mitochondrial homeostasis ${ }^{32,33}$, excessive mitochondrial mitophagy may promote cell death ${ }^{34}$. Our results showed the LC3-II/LC3-I ratio in ER-enriched fragments was about $4 / 1$ at $8 \mathrm{~h}$, but the ratio in Mito-enriched fragments was about $4 / 1$ at $24 \mathrm{~h}$ (Fig. 2e); there was no significant difference at $12 \mathrm{~h}$ (Supplementary Figure 2). PINK1 (PTEN-induced putative kinase1) functions in a common signaling pathway known to regulate mitochondrial network homeostasis and quality control, including mitophagy $^{35}$. The results showed that PINK1 siRNA significantly inhibited autophagy and cell apoptosis at $24 \mathrm{~h}$ post-OA treatment (Supplementary Figure 3). According to the guidelines, we detected colocalization of mitochondria or ER with lysosomes ${ }^{36}$. Our results showed that merged dots of ER with lysosomes peaked at $8 \mathrm{~h}$, but the 
A

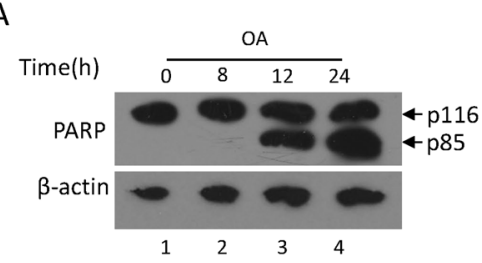

E Time (h)

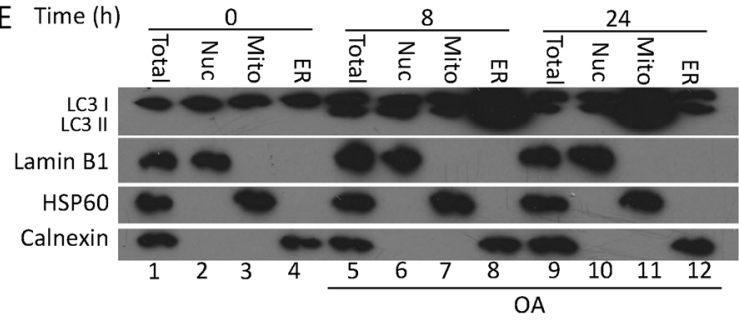

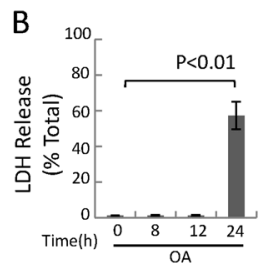
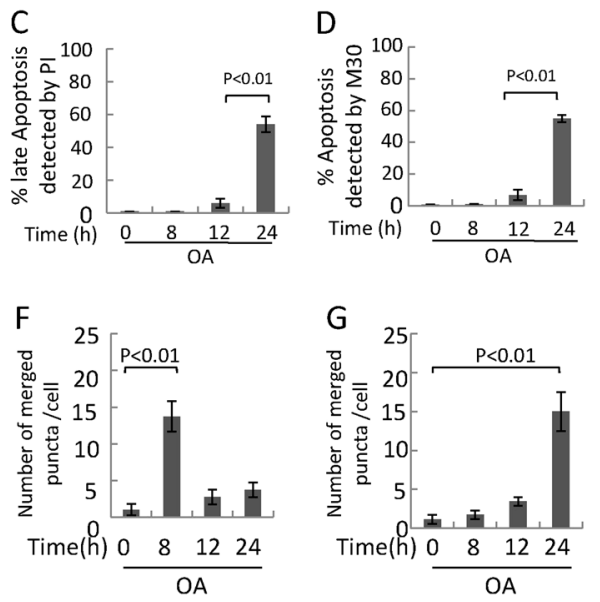

$\mathrm{H}$
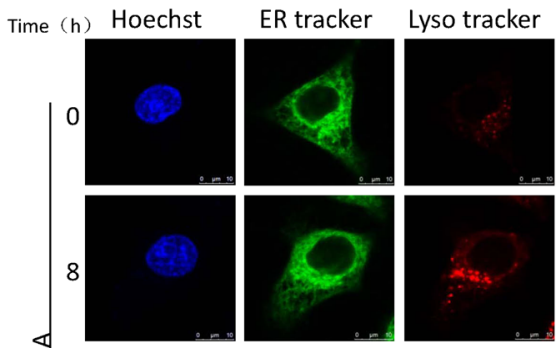

త
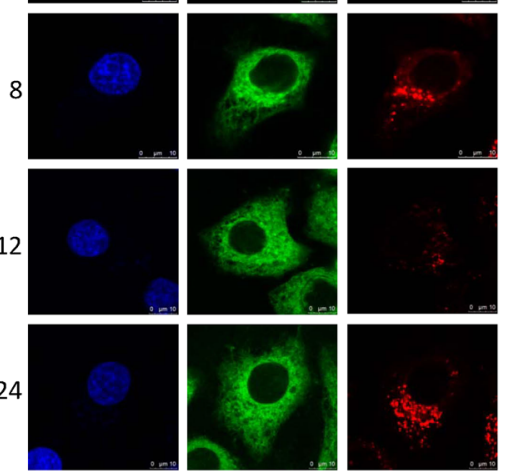

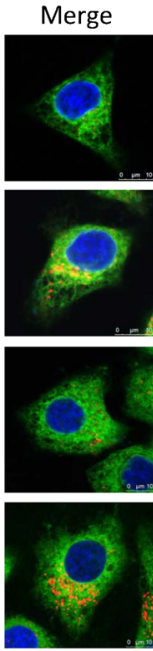

I
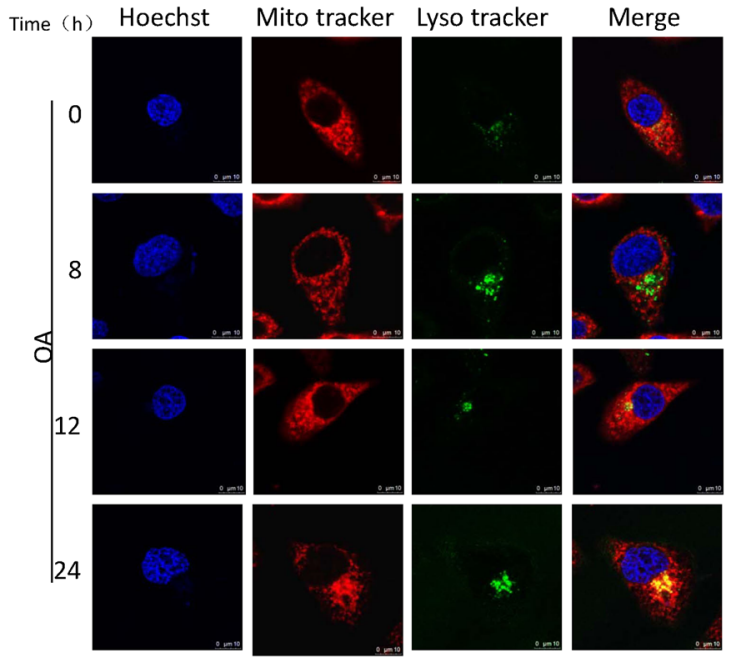

Fig. 2 OA treatment induces apoptosis, cell impairment, reticulophagy, and mitophagy development. HepG2 cells were treated with $400 \mu \mathrm{M} O \mathrm{~A}$ for $24 \mathrm{~h}$. a Representative western blotting analysis of cleaved PARP fragment in cells. b The levels of LDH release in supernatant. c, $\mathbf{d}$ Quantification of apoptotic cells by Calcein AM/PI(B) and M30(C) immunoreactivity. Data are presented as mean \pm SEM in three independent experiments. e Subcellular fractions of HepG2 cells were subjected to a western blotting assay with indicated antibodies. Lamin B1, HSP60, and calnexin were used as controls for the nuclei (Nuc), mitochondrial (Mito), and endoplasmic reticulum (ER) fractions, respectively. Total: total lysate of cells. $\mathbf{h}$ The degree of colocalization of endoplasmic reticulum with lysosomes in HepG2 cells was measured via live-cell imaging microscopy. LysoTracker Red DND-99 staining was applied to mark lysosomal structures (red), and ER-Tracker Green to visualize endoplasmic reticulum (green). Hoechst 33342 dye was used to stain nuclei (blue). A positive colocalization is indicated by yellow signals (merge) due to the overlap of LysoTracker Red and ER-Tracker Green staining. Scale bars: $10 \mu m$. i The degree of colocalization of mitochondria with lysosomes in HepG2 cells. LysoTracker was green pseudo, and MitoTracker was red. A positive colocalization is indicated by yellow signals (merge) due to the overlap of LysoTracker Red and MitoTracker Green staining. Scale bars: $10 \mu \mathrm{m}$. $\mathbf{f}, \mathbf{g}$ Quantification of the merged number which was yellow. Data are presented as mean \pm SEM in three independent experiments

merged dots of mitochondria with lysosomes peaked at $24 \mathrm{~h}$ (Fig. 2f-i). These results suggest that the first wave of autophagy occurred mainly in the ER, whereas the second wave occurred mainly in the mitochondria.

\section{The persistence of the first wave of autophagy} downregulates lipotoxicity and hepatocyte death induced by $O A$

Removing OA from the culture medium after $8 \mathrm{~h}$ of OA treatment, we observed that the levels of lipid droplets and intracellular triglycerides after $8 \mathrm{~h} \mathrm{OA}+24 \mathrm{~h}$ DMED returned to the baseline level (Fig. 3a). Moreover, we examined the effects of removing OA on autophagic flux. Following an 8-h pre-treatment with OA, autophagy was observed 12 and $24 \mathrm{~h}$ after the removal of OA (Fig. 3a, b), which coincided with a reduction in lipid droplet formation (Fig. 3a). According to western blotting, we found that LC3-II mainly exists in the ER-enriched fragments from 8 to $24 \mathrm{~h}$ after OA withdrawal (Fig. 3c), suggesting that the autophagic process initially consists mainly of 


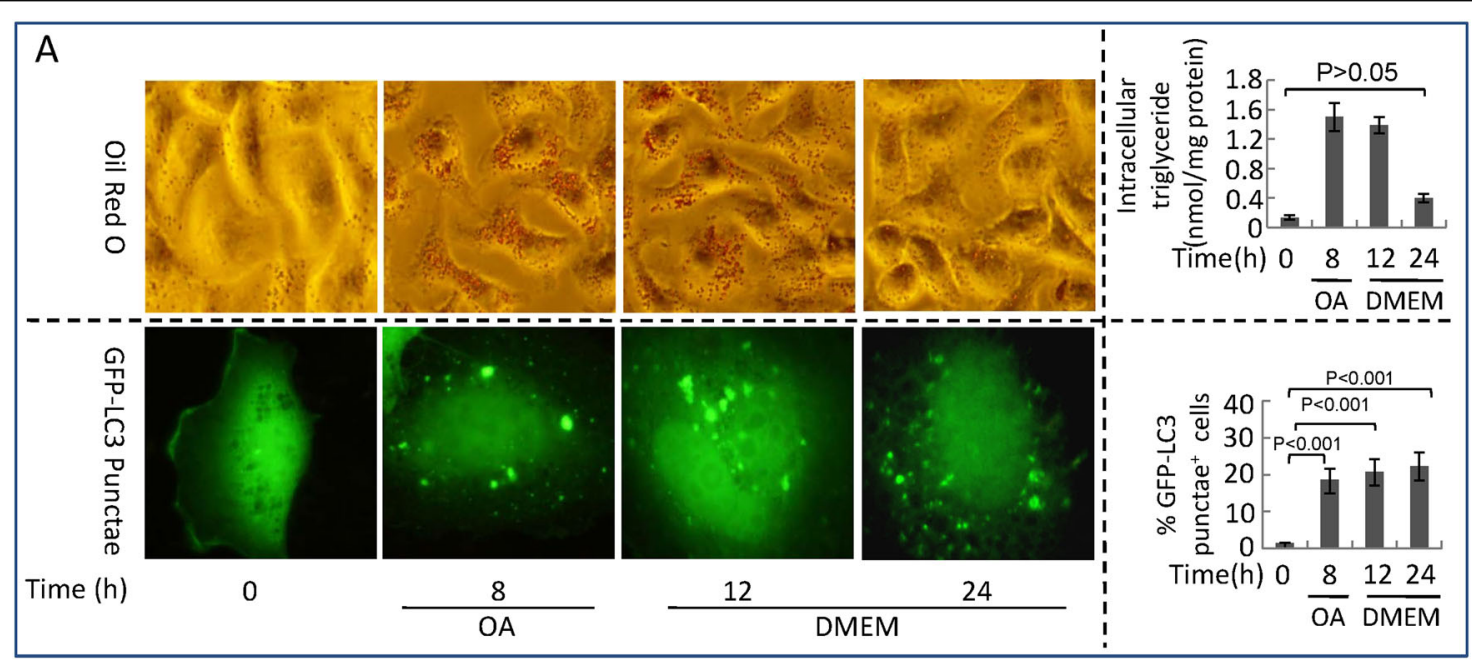

B

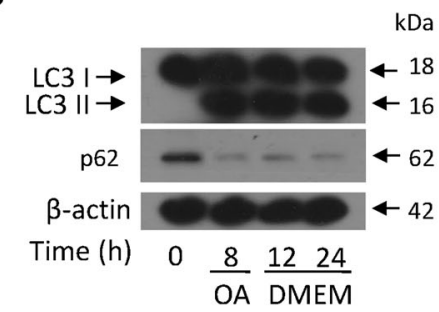

D

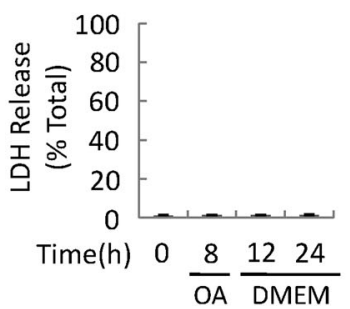

C Time (h)

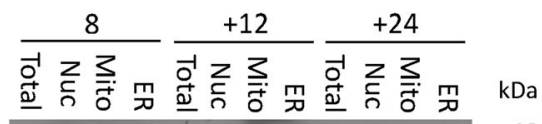

LC3

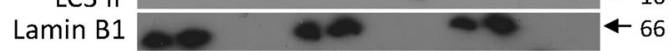

HSP60

Calnexin

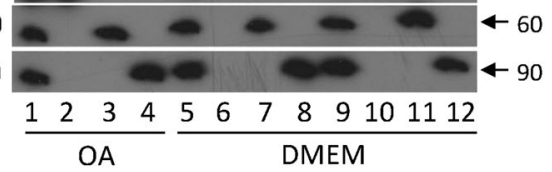

$E$

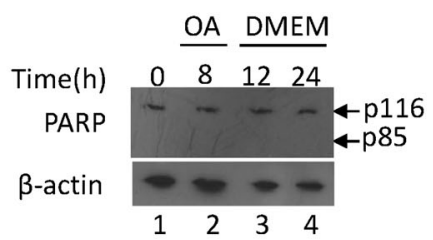

Fig. 3 The first wave of autophagy prevents $400 \mu \mathrm{M}$ OA-induced lipotoxicity and hepatocytic apoptosis. HepG2 cells were stimulated by 400 $\mu \mathrm{M} O \mathrm{O}$ for $8 \mathrm{~h}$ and then were cultured in normal DMEM culture medium for 12 and $24 \mathrm{~h}$. a Oil Red $\mathrm{O}$ staining (upper left panel, original magnification, $\times 400$ ) and intracellular triglyceride levels of cells (upper right panel); representative GFP-LC3 images (lower left panel, original magnification, $\times 1000$ ) and the percentage of autophagosomes (lower right panel) of the cells. Cells with 5 or more GFP-LC3 puncta were considered to have accumulated autophagosomes. Data are presented as mean \pm SEM in three independent experiments. $\mathbf{b}$, e Representative western blotting analysis of LC3 I/II and p62(B) and cleaved PARP fragment e. c Subcellular fractions were subjected to a western blotting assay with indicated antibodies. Lamin B1, HSP60, and calnexin were used as controls for the nuclei (Nuc), mitochondrial (Mito), and endoplasmic reticulum (ER) fractions, respectively. Total: total lysate of cells. $\mathbf{d}$ The levels of LDH release in HepG2 cells were analyzed. Data are presented as mean \pm SEM in three independent experiments

reticulophagy following the removal of OA. The persistence of reticulophagy did not induce bulk LDH release at $24 \mathrm{~h}$ (Fig. 3d) or the development of apoptosis at 12 and $24 \mathrm{~h}$ (Fig. 3e). These data suggest that OA removal extends the persistence of the reticulophagy-predominant phase to $24 \mathrm{~h}$. Importantly, OA withdrawal does not increase cell death and leads to a decrease in the level of lipid droplets, which suggests that reticulophagy during early ER stress is not associated with the induction of cell death. Based on this observation, we investigated the role of reticulophagy in protecting hepatocytes from lipotoxicity-induced apoptosis.

The first wave of reticulophagy upon OA treatment for $8 \mathrm{~h}$ might play a protective role associated with autophagy inhibition, but the second wave of mitophagy has opposite effects

To elucidate the relationship between the two waves of autophagy and the occurrence of cell death, we used LY294002 to inhibit autophagy. Our results showed that 
LY294002 pre-treatment induced increasing severe lipotoxicity (Fig. 4a-c). Calcein AM/PI and M30 immunoreactivity assays showed that cell death significantly increased from 8 to $12 \mathrm{~h}$ of OA treatment when PI3K was inhibited by LY294002 (Fig. 4e-h). The cleaved PARP fragment (p85) band could be detected upon OA treatment for $8 \mathrm{~h}$ (Fig. $4 \mathrm{~d}$ ). These results suggest that autophagy that occurs within the 8-h time frame post-OA treatment might play a protective role. However, the second wave of autophagy inhibited by LY294002 decreased LDH release, and cell death at $24 \mathrm{~h}$ of OA treatment suggests that the second wave of autophagy promotes apoptosis (Fig. 4c, e-h). Single agent LY294002 treatment at $50 \mu \mathrm{M}$ does not induce HepG2 cell death (Supplementary Figure 4). In addition, ATG5 siRNA significantly inhibited autophagy at 8 and $24 \mathrm{~h}$ post-OA treatment, and inhibited cell apoptosis distinctly at $24 \mathrm{~h}$ (Supplementary Figure 5).

\section{Activation of the PI3K/AKT signaling pathway is involved in reticulophagy; mitophagy induces HepG2 apoptosis by promoting DRAM expression and by decreasing $\mathrm{BCL}-2$ expression}

PI3K/AKT signaling pathway activation is associated with an anti-apoptotic function, and the inhibition of the PI3K/AKT pathway contributes to NAFLD development ${ }^{37}$. We sought to determine whether OA treatment could inhibit PI3K/AKT activation and induce apoptosis. Our results showed that phospho-PI3K and phospho-AKT could be detected at $8 \mathrm{~h}$ (Fig. 5a, column 3), BCL-2 expression increased at $8 \mathrm{~h}$ post-OA treatment (Fig. 5a, column 3), and BAX expression increased at 12 and $24 \mathrm{~h}$ (Fig. 5a, columns 5 and 7). Moreover, LY294002 inhibited the activation of the PI3K/AKT pathway, which is shown by the absence of phospho-PI3K and phospho-AKT $8 \mathrm{~h}$ post-OA treatment (Fig. 5a, column 4). Using VPS34 siRNA to inhibit autophagy, we obtained similar results to the LY294002-treated samples (Fig. 5b). Thus, our results suggest that the first wave of autophagy induces PI3K/AKT pathway activation. Furthermore, by removing $\mathrm{OA}$ after $8 \mathrm{~h}$ of treatment, our results show that BCL-2, phospho-PI3K, and phospho-AKT could still be detected 12 and $24 \mathrm{~h}$ after the initial exposure to OA (Fig. 5c). These results suggest that the PI3K/AKT pathway remains activated simultaneously with the first wave of autophagy.

\section{p53-dependent DRAM expression is involved in the second} wave of autophagy and contributes to HepG2 apoptosis

Previous studies have shown that an imbalance of BCL2 and BAX expression is involved in hepatocytic apoptosis during NAFLD. BAX is a transcriptional target of $\mathrm{p} 53^{38}$, and p53 can also regulate the expression of lipid metabolism-associated genes, the dysfunction of which can lead to NAFLD development ${ }^{39}$. The effect of p53 on autophagy is currently unclear: basal levels of p53 function directly in the cytoplasm to inhibit autophagy ${ }^{21}$, whereas some transcriptional targets of p53 (e.g., DRAM) promote autophagy, a response that is consistent with the tumor-suppressive role of $\mathrm{p} 53^{40}$. Thus, we tried to determine how the activation of p53 signaling contributes to the cellular decision to initiate autophagy or apoptosis. We found that mRNA levels of PUMA, p21, and DRAM increased $24 \mathrm{~h}$ post-OA treatment (Fig. 6a). DRAM contributes to cell death by inducing autophagy ${ }^{41}$. Our results showed that DRAM expression was significantly increased after $24 \mathrm{~h}$ OA treatment (Fig. 6).

To further elucidate the role of DRAM, we analyzed the effect of inhibiting DRAM on the progression of autophagy, and we found that DRAM siRNA significantly inhibited autophagy at $24 \mathrm{~h}$ but not at $8 \mathrm{~h}$ post-OA treatment (Fig. $7 \mathrm{a}-\mathrm{c}$ ). Considering that the second wave of autophagy was mainly composed of mitophagy (Fig. 2e), these results suggest that DRAM plays a key role in inducing mitophagy in HepG2 cells exposed to OA. In fact, our previous data showed that $400 \mu \mathrm{M}$ OA induced DRAM expression and promoted its mitochondrial localization after $24 \mathrm{~h}^{42}$. Interestingly, the PI positive and M30 immunoreactive cells were significantly reduced in DRAM siRNA-treated HepG2 cells at $24 \mathrm{~h}$ post-OA treatment (Fig. 7d), and the leakage of LDH decreased dramaticlly at 24 $\mathrm{h}$ (Fig. 7e). These results suggest that DRAM-dependent mitophagy cooperates with other p53-dependent apoptotic signals (e.g., PUMA, p21, BAX) to induce apoptosis.

Autophagy-dependent activation of the PI3K/AKT pathway is present in the liver tissue of patients with low-grade steatosis, and DRAM overexpression was observed in liver tissue of patients with high-grade steatosis

We observed an induction of autophagy in low-grade steatosis and high-grade steatosis patients (Fig. 8a). Upregulation of M30 has been shown to be associated with NALFD deterioration ${ }^{28-30}$, and our data further confirm this, as we observed increased expression of M30 and elevated mRNA levels of PUMA and p21 in the livers of high-grade steatosis group, suggesting that apoptosis is mainly induced in patients with high-grade steatosis (Supplementary Figure 6). Western blotting results showed that PI3K/AKT phosphorylation was present only in liver samples from patients with low-grade steatosis, and DRAM protein expression was higher in liver samples from patients with high-grade steatosis (Figs 7 and 8). These clinical results support our in vitro findings that autophagy is an independent sequence of events involved in the pathological progression of NAFLD, and autophagy plays different roles as an anti- and pro-apoptotic pathway.

\section{Discussion}

Previous reports have shown that autophagy is impaired in patients with NAFLD. Some factors, such as ER stress, 

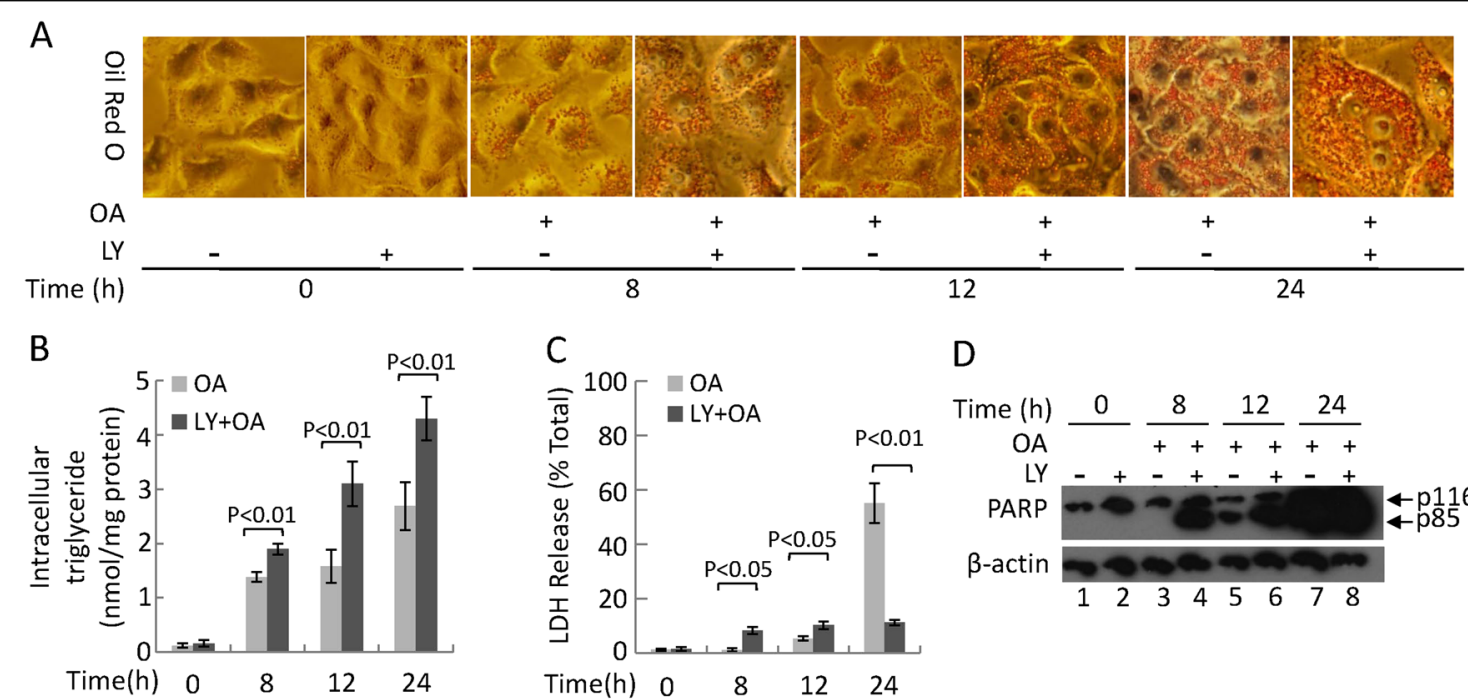

D

$\mathrm{E}$
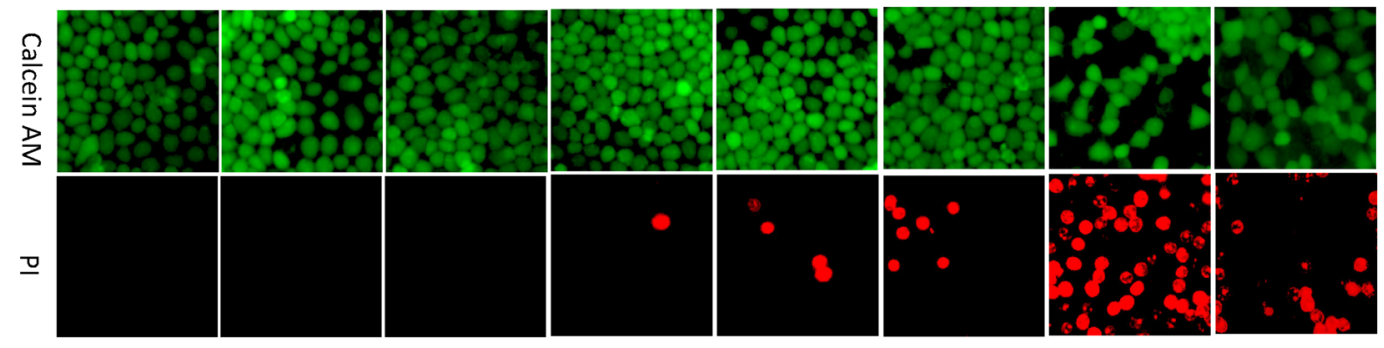

$\mathrm{F}$
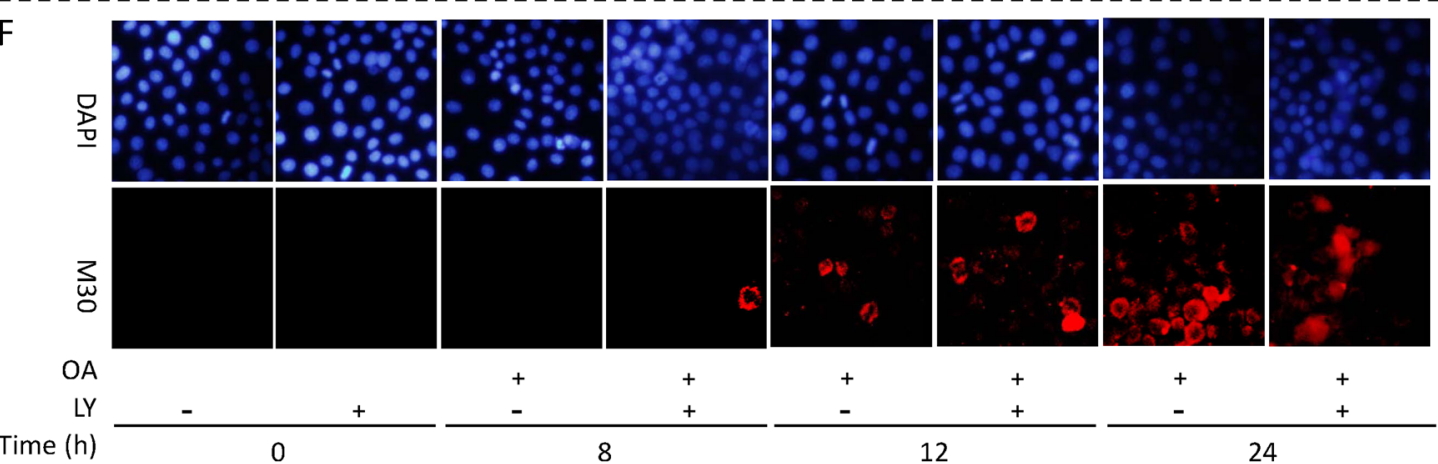

Time (h)

$\mathrm{OA}$

G
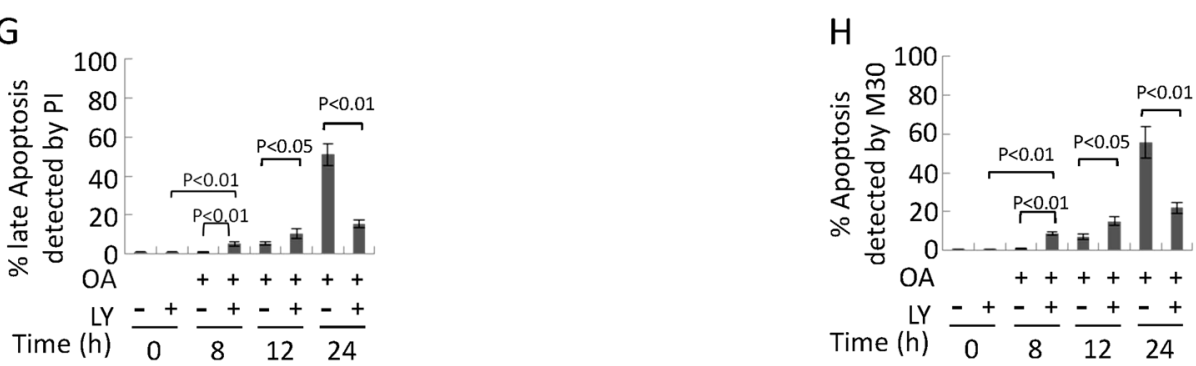

Fig. 4 Autophagy inhibition by LY294002 (LY) promotes earlier apoptosis development at $\mathbf{8} \mathrm{h}$, but decreases the level of apoptosis at 24 h. LY294002, a PI3K inhibiter, was added into the culture for $5 \mathrm{~h}$ to inhibit autophagy and replaced by $400 \mu \mathrm{M}$ OA. a Oil Red O staining (original magnification, $\times 400$ ). $\mathbf{b}$ Intracellular triglyceride levels. c The levels of LDH release. $\mathbf{d}$ Representative western blotting analysis of cleaved PARP fragment in cells. Representative images of calcein AM/PI staining (e), DAPI/M30 staining (f), and quantification of late (g) and early (h) apoptosis by PI staining and M30 immunoreactivity, respectively. $\mathbf{b}, \mathbf{c}, \mathbf{g}, \mathbf{h}$ Data are presented as mean \pm SEM in three independent experiments 

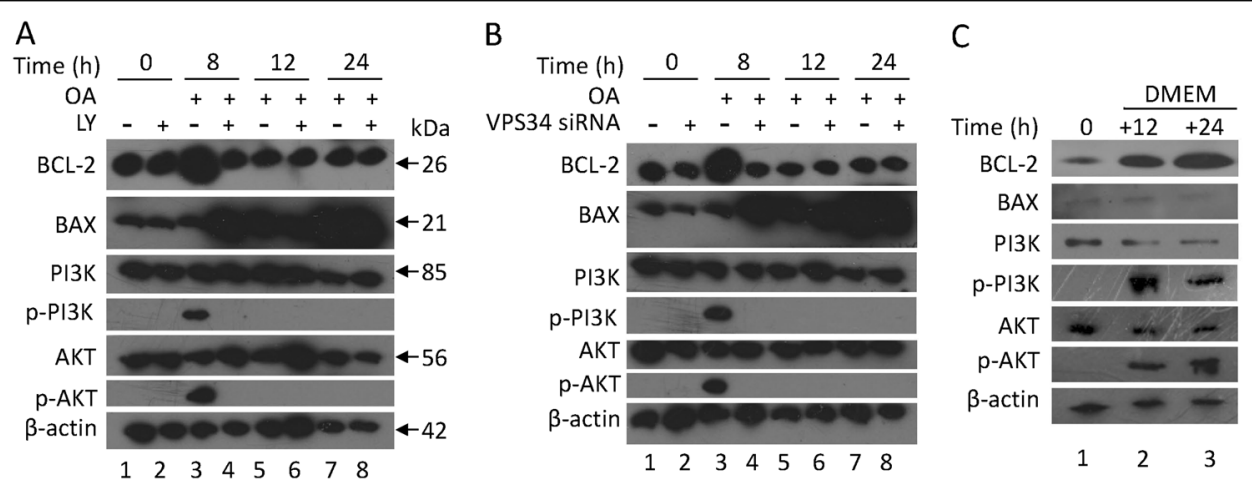

Fig. 5 OA treatment activates PI3K/AKT signaling pathway and increases BCL-2 expression at $8 \mathrm{~h}$, whereas increases BAX expression at $\mathbf{2 4}$ h. HepG2 cells were pre-treated with LY294002 (a) or VPS34 siRNA (b) and then were cultured with $400 \mu \mathrm{MM} \mathrm{OA.} \mathrm{c} \mathrm{HepG2} \mathrm{cells} \mathrm{were} \mathrm{treated} \mathrm{with}$ $400 \mu \mathrm{M}$ OA for $8 \mathrm{~h}$ and then were cultured in normal DMEM culture for 12 and $24 \mathrm{~h}$. a-c Lysates of HepG2 cells were collected, and an immunoblot assay was conducted with the indicated antibodies

insulin resistance, and mitochondrial dysfunction, have been shown to contribute to the deterioration of NAFLD, and associated with the impairment of autophagy in the context of NAFLD ${ }^{13}$. Thus, the impairment of autophagy appears to be associated with lipotoxicity and the deterioration of NAFLD. OA is a monounsaturated fatty acid, which presents in the diet and in blood plasma. It has been reported that OA had various effects, including stem cell modulation, apoptosis and autophagy, etc. ${ }^{43,44}$. The use of OA building NAFLD model has been successfully demonstrated in some studies; our results showed that reticulophagy and mitophagy were induced in HepG2 upon 8 and $24 \mathrm{~h}$ of $400 \mu \mathrm{M}$ OA treatment, respectively.

Autophagy has been regarded as a type of cell death distinguishable from apoptosis as well as a protective pathway that promotes cell survival ${ }^{45,46}$. Many past studies have focused on a single role of autophagy (initiation or prevention of cell death). Our study is unique in that we help to elucidate the anti- and pre-apoptotic functions of autophagy, which appear to be manifested through reticulophagy and mitophagy. The crosstalk always exists between autophagy and apoptosis, and components of the autophagic pathway may also directly function in the regulation of apoptosis ${ }^{47,48}$. In our study, reticulophagy is identified as a main factor involved in the inhibition of cell death by apoptosis, whereas mitophagy effects are contrary. We think this is a very interesting phenomenon, and may exist in other life processes. In many studies on NAFLD, lipotoxicity-induced ER stress exacerbates NAFLD and leads to the impairment of autophagy $y^{10,49}$. Mitophagy is a key factor for reducing the overproduction of reactive oxygen species (ROS), which is a potential inducer of apoptosis ${ }^{32,50}$.

However, our results elucidate previously unknown roles for reticulophagy and mitophagy in the progression of NAFLD. Lipotoxicity-induced reticulophagy appears to primarily prevent hepatocytes from apoptosis; however, continued FFA stimulus appears to prevent reticulophagy from rescuing hepatocytes from lipotoxicity, which leads to an increase in mitophagy. Subsequently, mitophagy promotes the development of hepatocytic apoptosis. It is possible to imply that reticulophagy and mitophagy play a protective or disadvantageous role, respectively, in the NAFLD progression. Previous studies reported that proand anti-apoptotic members of the BCL-2 family exert their activities not only at mitochondria but also at the $\mathrm{ER}^{51,52}$. According to some studies, mitochondria that undergo permeability transition (PT) with a loss of $\Delta \Psi_{\mathrm{m}}$ are preferentially targeted by mitophagy ${ }^{53}$. Thus, an attractive explanation for our observation is that OAinduced reticulophagy promotes PT-mediated mitophagy development via releasing calcium. However, the exact mechanism by which reticulophagy can be transformed into mitophagy is still unknown.

With respect to the "multiple-hit" hypothesis, lipid accumulation in hepatocytes is sufficient to induce detrimental factors that impair liver function ${ }^{54}$. The levels of lipid droplets and intracellular triglycerides at $8 \mathrm{~h}$ of $\mathrm{OA}$ treatment significantly increase relative to the basal levels but are significantly less at $24 \mathrm{~h}$, and our results did not show that lipophagy promotes the downregulation of lipotoxicity induced by OA treatment. However, our results showed that reticulophagy is associated with the disappearance of lipid droplets and intracellular triglycerides after OA treatment. An attractive explanation for our observation is that reticulophagy regulates ER homeostasis during the unfolded protein response (UPR), and also reduces triglyceride formation by degrading dysfunctional ER. These results suggest that the different types of autophagy may not involve in the same process and function. Moreover, the findings that the liver tissue of patients with low-grade steatosis accumulates less lipid suggest that autophagy may regulate lipid metabolism 

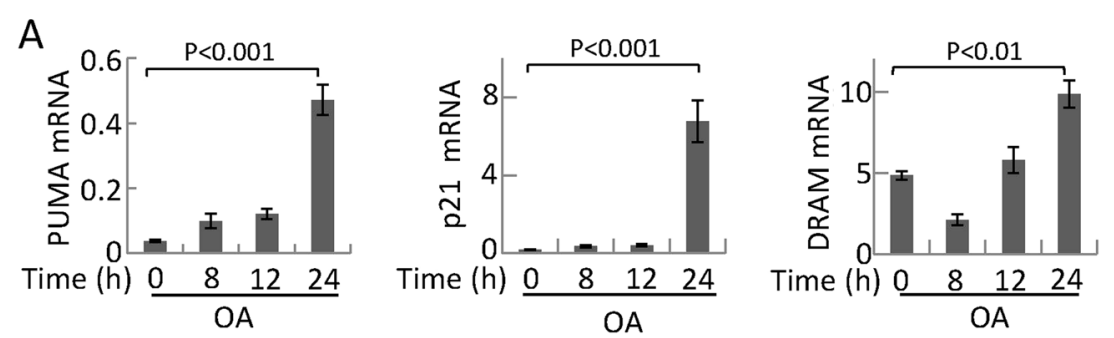

B

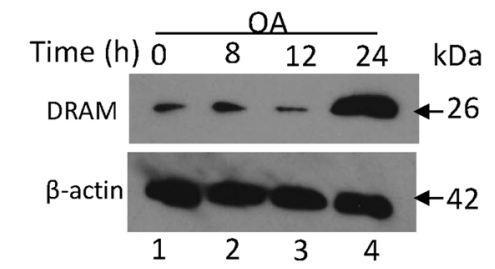

Fig. 6 p53 signaling pathway was activated by $400 \mu \mathrm{M}$ OA treatment. a Total RNA was isolated from HepG2 cells treated with $400 \mu \mathrm{M}$ OA. Realtime PCR was employed to detect the mRNA level of PUMA, p21, and DRAM. b Lysates of HepG2 cells were collected, and an immunoblot assay was conducted with the anti-DRAM antibody

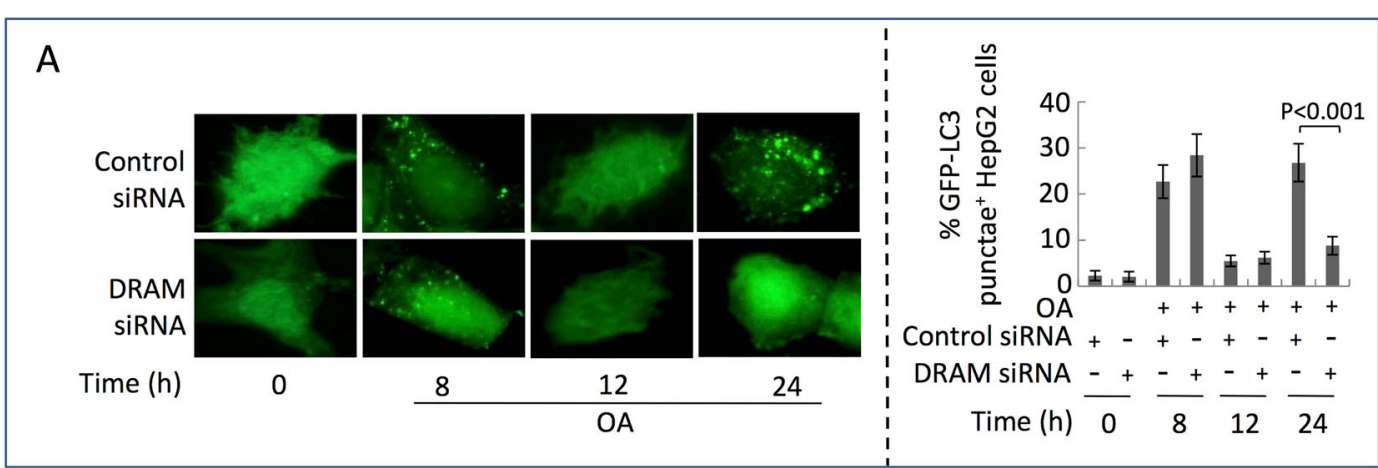

B
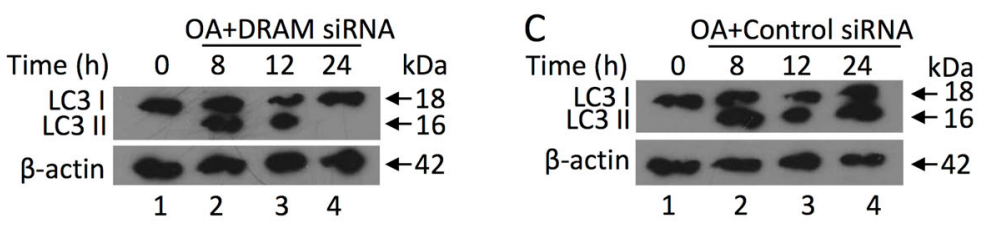

D
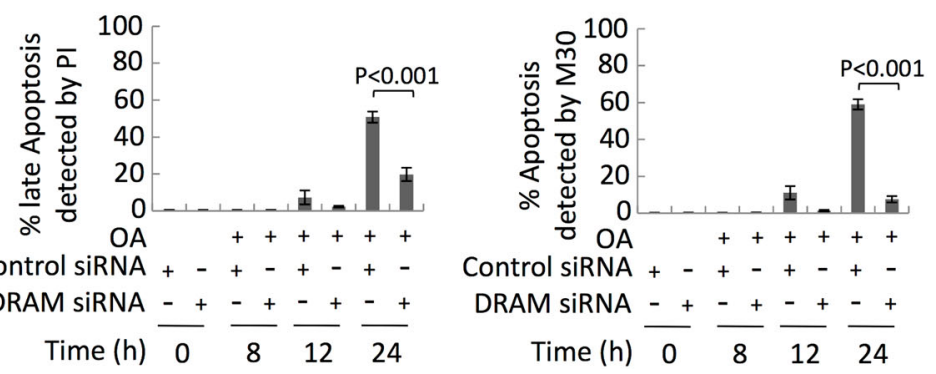

$E$

Fig. 7 Following $400 \mu \mathrm{M}$ OA treatment, DRAM expression induced the second wave of autophagy. HepG2 cells were pre-treated with DRAM siRNA and then cultured with $400 \mu \mathrm{M}$ OA. a Representative GFP-LC3 images (left panel, original magnification, $\times 1000$ ), quantification of autophagosome formation (right panel). b, c Lysates of HepG2 cells were collected, and an immunoblot assay was conducted with the indicated antibodies. d Quantification of apoptotic cells by calcein AM/PI and M30 immunoreactivity. e The levels of LDH release. a, d, e Data are presented as mean \pm SEM in three independent experiments 


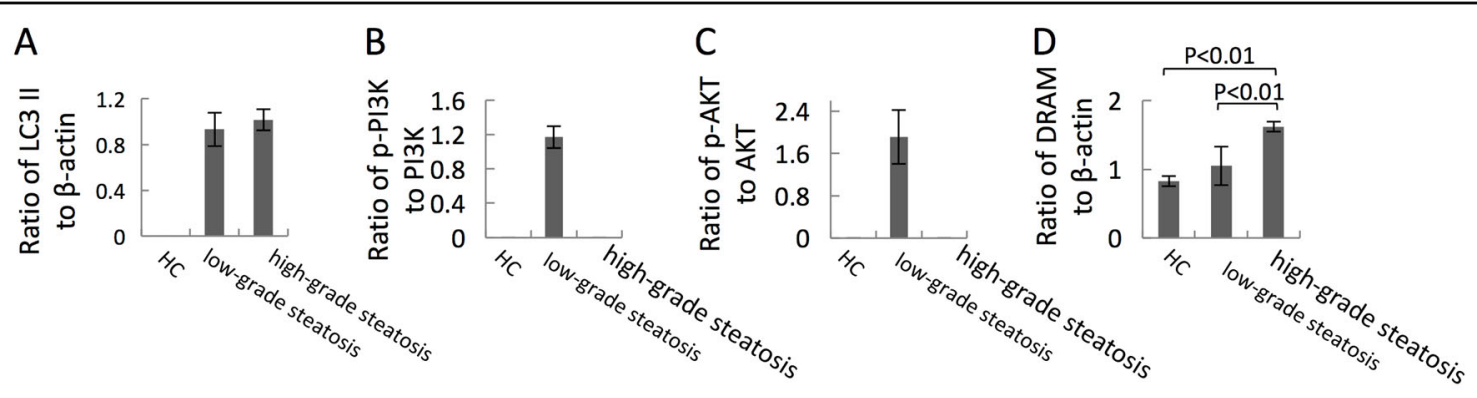

E

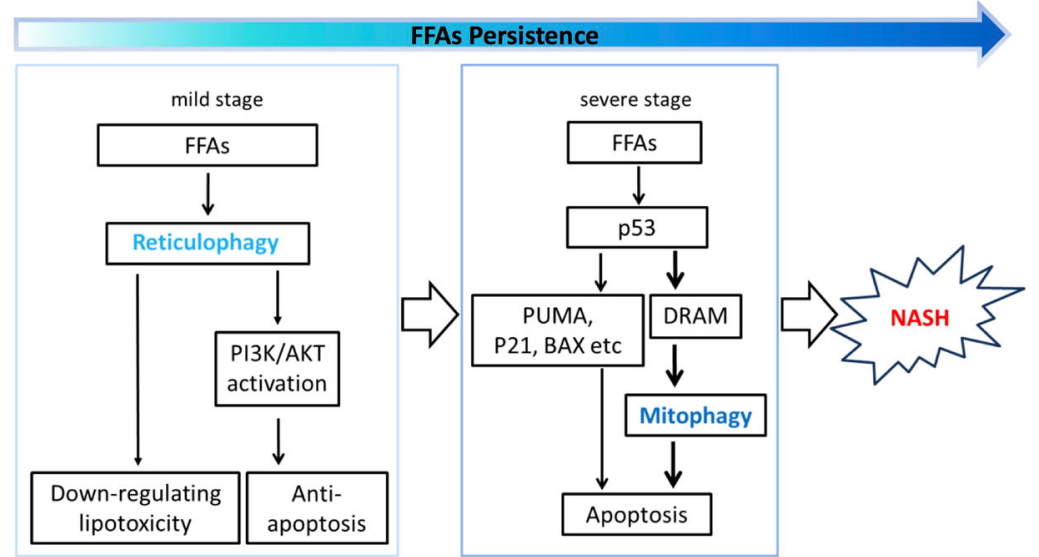

Fig. 8 Autophagy development, activation of PI3K/AKT, and DRAM signaling pathways were detected in liver tissues of patients with lowgrade steatosis and high-grade steatosis. Lysates of liver tissues were collected and immunoblot assay was conducted with the indicated antibodies. Densitometry analysis of the band density ratio of LC3 II (a)/DRAM (d) to $\beta$-actin and phosphor-PI3K (b)/-AKT (c) to their non-active forms. e Model. In the mild stage of NAFLD, FFAs primarily induce reticulophagy to protect hepatocytes via downregulating lipotoxicity and activating the PI3K/AKT signaling pathway. In the severe stage when FFAs are persistent, FFAs induce DRAM expression through p53 and causes hepatocytic apoptosis, through mitophagy in concert with one or more p53-dependent apoptotic signals, leading to NASH development

during the mNAFLD stage. However, the subtype of autophagy that regulates lipid metabolism in liver tissues of NAFLD patients remains unknown.

In addition to decreasing lipotoxicity, reticulophagy has been associated with the activation of the PI3K/AKT pathway. Some studies have demonstrated that during the development of NAFLD, autophagy is inhibited by the PI3K/AKT signaling pathway via both short-term and long-term regulation mechanisms ${ }^{13}$. However, our results reveal a new relationship between PI3K/AKT signaling pathway activity, reticulophagy, and NAFLD development. Our results suggest that the persistence of reticulophagy in early ER stress is dependent on PI3K/AKT pathway activation. $\mathrm{AKT}$ is phosphorylated by $\mathrm{PI} 3 \mathrm{~K}$ and becomes activated, and phospho-AKT can bind and regulate many downstream effectors such as BCL-2 family proteins ${ }^{55}$. $\mathrm{PI} 3 \mathrm{~K} / \mathrm{AKT}$ activation may promote $\mathrm{BCL}-2$ expression in reticulophagy and may play a protective role during the early stages of NAFLD development.

p53, a recognized tumor suppressor protein, acts as a master regulator with pleiotropic effects on metabolism, anti-oxidant defense, proliferation, senescence, and cell death $^{56}$. Previous data have shown that p53 activation is involved in cell apoptosis during NAFLD ${ }^{57}$, and regulates the balance between $\mathrm{BCL}-2$ and $\mathrm{BAX}^{58}$. In this study, our results demonstrate that $\mathrm{p} 53$ signaling is active during later stages of OA treatment. The p53 target genes were upregulated in HepG2 cells cultured with OA for 24h. p21 mediates p53-dependent cell cycle arrest, and PUMA induces apoptosis via interactions with BCL-2 family members ${ }^{59}$.

DRAM is critical for apoptosis, but it has rarely been reported in NAFLD. In this study, the results show that DRAM-mediated autophagy promotes apoptosis, and DRAM expression is only upregulated in the liver tissues of patients with high-grade steatosis. Thus, our data establish a link between p53 activation and mitophagy-mediated apoptosis in hepatocytes via DRAM expression. Because DRAM-mediated mitophagy cannot completely account for the cell apoptosis, we postulate that p21 and PUMA also play roles in inducing hepatocytic apoptosis. Thus, DRAMmediated autophagy appears to be a critical but not sufficient inducer of apoptosis when p53 is activated in NAFLD.

Taken together, our data suggest that reticulophagy and mitophagy are two independent events during NAFLD pathogenesis (Fig. 8e). Considering the distinct 
mechanisms and functions displayed by the two waves of autophagy, the different functions of various types of autophagy may offer new insights into the prevention and cure of NAFLD.

\section{Materials and methods}

\section{Cell culture and treatment}

The human hepatoblastoma cell line HepG2 was grown in Dulbecco's Modified Eagle's Medium (DMEM) (Invitrogen, Carlsbad, CA) supplemented with $10 \%$ fetal bovine serum (FBS) (Invitrogen, Carlsbad, CA). Oleic acid (Sigma-Aldrich, St. Louis, MO) was conjugated to albumin, and cells were treated with $400 \mu \mathrm{M}$ oleic acid for 24 h. BafA1 ( 50 nM, Sigma Inc., St. Louis, MO, USA) was used to inhibit the autophagy flux. PI3K inhibitor LY294002 $(50 \mu \mathrm{M})$ (Cell Signaling Technology, Beverly, MA) was used to inhibit autophagy by pre-treating cells for $5 \mathrm{~h}$. HepG2 cells were transfected with Fugene HD (Promega, Madison, WI) for the transfection of plasmids encoding GFP-LC3 or with Lipofectamine 2000 (Invitrogen, Carlsbad, CA) for the transfection of DRAM siRNA and control siRNA (the sequences of the siRNA were obtained from ref. $^{38}$ ), VPS34 siRNA (ACGGTGATGAATCATCTCCAA), ATG5 SiRNA (GAAGUUUGUCCUUCUGCU), or PINK1 siRNA (CCTCGTTA TGAAGAACTAT) (the sequences of the siRNA were obtained from refs. ${ }^{60,61}$. Cells grown on glass cover slips were used for immunofluorescence detection.

\section{Real-time PCR}

The RNeasy Mini Kit (Qiagen, Hilden, Germany) was used to isolate total RNA from cultured cells with or without treatment with OA and/or LY294002. Reverse transcription was conducted using the SuperScript II Firststrand Synthesis System for RT-PCR (Invitrogen, Carlsbad, CA) to synthesize first-strand cDNA. SYBR Green was used to detect dsDNA product during the real-time PCR reaction. The mRNA content was normalized to the housekeeping gene $\beta$-actin. Specific primer sequences used for real-time PCR were as follows: for PUMA, 5'-CGACCTCAACGCACAGTACGAG-3' (forward) and 5'-AGGA GTCCCATGATGAGATTGTACA-3' (reverse); for p21, 5'CAGGCTGAAGGGTCCCCAGGTGGA-3' (forward) and 5'-GGATTAGGGCTTCCTCTTGGAGA-3' (reverse); for DRAM, 5'-TCAAATATCACCATTGATTTCTGT-3' (forward) and 5'-GCCACATACGGATGGTCATCTCTG-3' (reverse) (the sequences of DRAM primers were obtained from ref. $\left.{ }^{38}\right)$; for $\beta$-actin, $5^{\prime}$-GCCCTGAGGCACTCTTCCA$3^{\prime}$ (forward) and 5'-CGGATGTCCACGTCACACTT-3' (reverse).

\section{Subcellular fractionation and western blotting}

Subcellular fractionation of HepG2 cells was conducted as described previously ${ }^{51}$. Briefly, the lysates of
HepG2 cells were centrifuged at $1000 \times g$ for $30 \mathrm{~min}$ and the pellet was considered nuclei-enriched fragment. The nuclei-depleted supernatant was centrifuged at 10,000 $\times$ $g$ for $30 \mathrm{~min}$ and the pellet was considered mitochondriaenriched fragment. The mitochondria-depleted supernatant was further centrifuged at $100,000 \times g$ for $2 \mathrm{~h}$ to yield the ER-enriched pellet. Lysates of cells and liver tissues were subjected to western blot analysis, as previously described ${ }^{34}$. Briefly, total cellular lysates were separated by $10 \%$ or $12 \%$ SDS-PAGE and then were transferred to PVDF membranes. The membranes were blocked with $5 \%$ milk, and were probed sequentially with specific primary antibodies and horseradish peroxidaseconjugated secondary antibodies (all antibodies were purchased from Santa Cruz Biotechnology (Santa Cruz, CA, USA) except anti-actin antibody (Sigma-Aldrich, St. Louis, MO)).

\section{Fluorescence microscopy and apoptosis assay}

Frozen cells were fixed with $10 \%$ paraformaldehyde/ PBS, incubated in $1 \%$ Triton X-100/PBS for $5 \mathrm{~min}$, blocked with $3 \%$ BSA/PBS, and probed with mouse anti-M30 antibody which was produced by our laboratory and is used for detecting early apoptosis ${ }^{62}$. Cy3-conjugated anti-mouse secondary antibodies (Sigma-Aldrich, St. Louis) were used to amplify the signal. Nuclei were counterstained with 4,6-diamidino2-phenylindole (DAPI) (Thermo Fisher, Eugene, Oregon, USA). Some cells were rinsed with $1 \times$ PBS once and then were incubated for $15 \mathrm{~min}$ with propidium iodide (PI) and calcein acetoxymethyl ester (calceinAM) (Invitrogen, Carlsbad, CA) to detect cells in the late stage of apoptosis. M30 immunoreactivity and PI staining were detected using a fluorescence microscope (Nikon Eclipse 80i). Quantitative apoptosis analysis was performed by counting more than 1000 cells in each sample.

\section{Live-cell imaging}

Detection and colocalization of mitochondria with lysosomes or ER with lysosomes was done. HepG2 cells were cultured on coverslips inside a Petri $3.5 \mathrm{~cm}$ dish. When cells have reached the desired confluence, remove the medium from the dish and add the pre-warmed $\left(37^{\circ}\right.$ C) probe-containing medium. Incubate $\left(37^{\circ} \mathrm{C}, 5 \% \mathrm{CO}_{2}\right)$ the cells for $30 \mathrm{~min}$, then replace the loading solution with a fresh medium and observe the cells using a confocal microscope (Leica TCS SP8, Germany) fitted with the correct filter set. ER-Tracker Green (BODIPY FL glibenchamide), LysoTracker Red DND-99, and MitoTracker Deep Red FM were purchased from Thermo Fisher (Eugene, Oregon, USA). We used working concentrations of $1 \mu \mathrm{M}, 75 \mathrm{nM}$, and $400 \mu \mathrm{M}$ for ER-Tracker, LysoTracker, and MitoTracker, respectively. 


\section{LDH assay}

The level of LDH in the supernatant of cultured cells was detected using an LDH assay kit (Applygen Technologies Inc., Beijing, China) according to the manufacturer's recommended protocol ${ }^{63}$.

\section{Intracellular triglyceride assay}

The concentration of intracellular triglycerides was estimated using an ultrasensitive assay kit for FFAs (Applygen Technologies Inc., Beijing, China) according to the manufacturer's recommended protocol ${ }^{63}$.

\section{Oil Red 0 staining}

HepG2 cells were washed twice with $1 \times$ PBS and were fixed with $4 \%$ paraformaldehyde for $15 \mathrm{~min}$. After three washes with PBS, cells were stained for $30 \mathrm{~min}$ in a freshly diluted Oil Red O solution. The dishes were rinsed in $1 \times$ PBS and then representative images of lipid droplets stained with Oil Red O were obtained by microscopic imaging.

\section{Patients and immunohistochemistry}

Patients with low-grade steatosis $(n=6)$ and highgrade steatosis $(n=6)$ NAFLD were recruited from You'an Hospital in Beijing. Normal liver tissues were obtained from resected liver metastases, which were used as healthy controls (HC, $n=4)$. The patients were diagnosed NAFL and excluded NASH via pathological and serological diagnosis. Our hospital doctors distinguished low-grade steatosis from high-grade steatosis by type-B ultrasonic and HE staining of the pathological section; steatosis less than 30\% was considered as low-grade steatosis and greater than $60 \%$ as high-grade steatosis. Liver tissues were fixed in $10 \%$ paraformaldehyde and then were embedded in paraffin. A 4- $\mu$ m-thick section cut from a paraffin-embedded block was stained with mouse monoclonal antibodies specific for M30. Briefly, liver sections were deparaffinized in xylene and were rehydrated in graded ethanol. The tissues were preincubated with $3 \%$ horse serum for $30 \mathrm{~min}$ to prevent non-specific antibody binding. Primary antibodies were diluted at 1:250 and were used to stain the tissues. The proteins were extracted from the paraffin-embedded liver tissues. Our protocol was approved by the ethics committee of You'an Hospital in Beijing, and all patients provided the informed consent for participation in this study.

\section{Statistical analysis}

All data represent at least three independent experiments and are expressed as the mean \pm SEM. Differences between the groups were compared using the Student's $t$-test or ANOVA. Differences were considered significant if the $p$-value was less than 0.05 .

\section{Acknowledgements}

This work was supported by National Natural Science Foundation of China (81272266), the Capital Health Development Research Projects (Capital Development 2014-1-1151), the Project of Construction of Innovative Teams and Teacher Career Development for Universities and Colleges under Beijing Municipality (IDHT20150502), Beijing Municipal Institute of Public Medical Research Development and Reform Pilot Project (2016-2), and Beijing science and technology planning project: Z141100002114002

\section{Author details}

'Beijing Institute of Hepatology, Capital Medical University, 100069 Beijing, China. ${ }^{2}$ Beijing You'an Hospital, Capital Medical University, 100069 Beijing, China. ${ }^{3}$ Department of Clinical Laboratory, Haidian Maternal \& Child Health Hospital, 100080 Beijing, China. ${ }^{4}$ Organ Transplantation Center, The Affiliated Hospital of Qingdao University, 26603 Qingdao, Shandong, China

\section{Conflict of interest}

The authors declare that they have no conflict of interest.

\section{Publisher's note}

Springer Nature remains neutral with regard to jurisdictional claims in published maps and institutional affiliations.

\section{Supplementary information}

The online version of this article https://doi.org/10.1038/s41419-017-0136-y contains supplementary material.

Received: 23 January 2017 Revised: 9 November 2017 Accepted: 9 November 2017

Published online: 24 January 2018

\section{References}

1. Wallace, M. B., Aqel, B. A., Lindor, K. D. \& Devault, K. R. Practical Gastroenterology and Hepatology Board Review Toolkit, Ch. 83 (John Wiley \& Sons Ltd., Oxford 2016).

2. Yki-Järvinen, $H$. Non-alcoholic fatty liver disease as a cause and a consequence of metabolic syndrome. Lancet Diabetes Endocrinol. 2, 901-910 (2014).

3. Hall, Z. et al. Lipid zonation and phospholipid remodeling in nonalcoholic fatty liver disease. Hepatology 65, 1165-1180 (2017).

4. Machado, M. V. \& Diehl, A. M. Pathogenesis of nonalcoholic steatohepatitis. Gastroenterology 150, 1769-1777 (2016)

5. Tilg, H. \& Moschen, A. R. Evolution of inflammation in nonalcoholic fatty liver disease: the multiple parallel hits hypothesis. Hepatology 52, 1836-1846 (2010).

6. Lim, J. S., Mietussnyder, M., Valente, A., Schwarz, J. M. \& Lustig, R. H. The role of fructose in the pathogenesis of NAFLD and the metabolic syndrome. Nat. ReV. Gastroenterol. Hepatol. 7, 251-264 (2010).

7. Singh, R. et al. Autophagy regulates lipid metabolism. Nature 458, 1131-1135 (2009).

8. Deegan, S., Saveljeva, S., Gorman, A. M. \& Samali, A. Stress-induced self-cannibalism: on the regulation of autophagy by endoplasmic reticulum stress. Cell. Mol. Life Sci. 70, 2425-2441 (2013).

9. Sica, V. et al. Organelle-specific initiation of autophagy. Mol. Cell 59, 522-539 (2015).

10. Greenberg, A. S. et al. The role of lipid droplets in metabolic disease in rodents and humans. J. Clin. Invest. 121, 2102-2010 (2011).

11. Booth, L. A., Tavallai, S., Hamed, H. A., Cruickshanks, N. \& Dent, P. The role of cell signalling in the crosstalk between autophagy and apoptosis. Cell. Signal. 26, 549-555 (2014).

12. Cully, M., You, H., Levine, A. J. \& Mak, T. W. Beyond PTEN mutations: the PI3K pathway as an integrator of multiple inputs during tumorigenesis. Phys. Chem. Chem. Phys. 12, 6309-6029 (2010).

13. Rautou, P. E. et al. Autophagy in liver diseases. J. Hepatol. 53, 1123-1134 (2010).

14. Derdak, Z. et al. Inhibition of p53 attenuates steatosis and liver injury in a mouse model of non-alcoholic fatty liver disease. J. Hepatol. 58, 785-791 (2013). 
15. Shen, W. et al. A novel and promising therapeutic approach for NSCLC: recombinant human arginase alone or combined with autophagy inhibitor. Cell Death Dis. 8, e2720 (2017).

16. Leibowitz, B. J. et al. Uncoupling p53 functions in radiation-induced intestinal damage via PUMA and p21. Mol. Cancer Res. 9, 616-625 (2011).

17. Zhang, L. et al. Pazopanib, a novel multi-kinase inhibitor, shows potent antitumor activity in colon cancer through PUMA-mediated apoptosis. Oncotarget 8, 3289-3303 (2017).

18. Masahiro, T. et al. Overexpression of DRAM enhances p53-dependent apoptosis. Cancer Med. 2, 1-10 (2013).

19. Guan, J. J. et al. DRAM1 regulates apoptosis through increasing protein levels and lysosomal localization of BAX. Cell Death Dis. 6, e1624 (2015).

20. Maiuri, M. C. et al Stimulation of autophagy by the p53 target gene Sestrin2. Cell Cycle 8, 1571-1576 (2009).

21. Tang, J., Di, J., Cao, H., Bai, J. \& Zheng, J. p53-mediated autophagic regulation: a prospective strategy for cancer therapy. Cancer Lett. 363, 101-107 (2015).

22. Ueno, T. \& Komatsu, M. Autophagy in the liver: functions in health and disease. Nat. Rev. Gastroenterol. Hepatol. 14, 170-184 (2017).

23. Zhu, C. et al. Mechanism of the promotion of steatotic HepG2 cell apoptosis by cholesterol. Int. J. Clin. Exp. Pathol. 7, 6807-6813 (2014)

24. Cui, W., Chen, S. L. \& Hu, K. Q. Quantification and mechanisms of oleic acidinduced steatosis in HepG2 cells. Am. J. Transl. Res. 2, 95-104 (2010).

25. Ricchi, M. et al. Differential effect of oleic and palmitic acid on lipid accumulation and apoptosis in cultured hepatocytes. J. Gastroenterol. Hepatol. 24, 830-840 (2009).

26. Mizushima, N., Yoshimori, T. \& Levine, B. Methods in mammalian autophagy research. Cell 140, 313-326 (2010).

27. Pandit, B. \& Gartel, A. L. Proteasome inhibitors induce p53-independent apoptosis in human cancer cells. Am. J. Pathol. 178, 355-360 (2011).

28. Younossi, Z. M. et al. A biomarker panel for non-alcoholic steatohepatitis (NASH) and NASH-related fibrosis. Obes. Surg. 21, 431-439 (2011).

29. Shen, J. et al. Non-invasive diagnosis of non-alcoholic steatohepatitis by combined serum biomarkers. J. Hepatol. 56, 1363-1370 (2012)

30. Guo, H. et al. Effects of bayberry juice on inflammatory and apoptotic markers in young adults with features of non-alcoholic fatty liver disease. Nutrition $\mathbf{3 0}$, 198-203 (2014)

31. Khaminets, A. et al. Regulation of endoplasmic reticulum turnover by selective autophagy. Nature 522, 354-358 (2015).

32. Mendl, N. et al. Mitophagy in yeast is independent of mitochondrial fission and requires the stress response gene WHI2. J. Cell Sci. 124, 1339-1350 (2011).

33. Hollville, E., Carroll, R. G., Cullen, S. P. \& Martin, S. J. Bcl-2 family proteins participate in mitochondrial quality control by regulating Parkin/PINK1dependent mitophagy. Mol. Cell 55, 451-46 (2014).

34. Kim, I., Rodriguezenriquez, S. \& Lemasters, J. J. Selective degradation of mitochondria by mitophagy. Arch. Biochem. Biophys. 462, 245-53 (2007).

35. McWilliams, T. G. \& Muqit, M. M. PINK1 and Parkin: emerging themes in mitochondrial homeostasis. Curr. Opin. Cell Biol. 45, 83-91 (2017).

36. Klionsky, D. J. et al. Guidelines for the use and interpretation of assays for monitoring autophagy (3rd edition). Autophagy 12, 1-222 (2016).

37. Han, J. W. et al. Impaired PI3K/Akt signal pathway and hepatocellular injury in high-fat fed rats. World J. Gastroenterol. 16, 6111-6118 (2010).

38. Follis, A. V. et al. Pin1-Induced Proline Isomerization in Cytosolic p53 Mediates BAX Activation and Apoptosis. Mol. Cell 59, 677-684 (2015).

39. Goldstein, I. et al. p53, a novel regulator of lipid metabolism pathways. BMC Proc. 56, 656-662 (2012)

40. Crighton, D. et al. DRAM, a p53-induced modulator of autophagy, is critical for apoptosis. Cell 126, 121-134 (2006)
41. Farrell, G. C. et al. Apoptosis in experimental NASH is associated with p53 activation and TRAIL receptor expression. J. Gastroenterol. Hepatol. 24, 443-452 (2009).

42. Liu, K. et al Depending on the stage of hepatosteatosis, p53 causes apoptosis primarily through either DRAM-induced autophagy or BAX. Liver Int. 33, 1566-1574 (2013).

43. Cardoso GB et al. Fatty acid is a potential agent for bone tissue induction: in vitro and in vivo approach. Exp. Biol. Med. 1013789728, 1-7 (2017).

44. Jiang, L. et al. Oleic acid induces apoptosis and autophagy in the treatment of Tongue Squamous cell carcinomas. Sci. Rep. 7, 11277 (2017).

45. Levine, B. \& Kroemer, G. Autophagy in the pathogenesis of disease. Cell 132 27-42 (2008).

46. Gordy, C. \& He, Y. W. The crosstalk between autophagy and apoptosis: where does this lead? Protein Cell 3, 17-27 (2012).

47. Gump, J. M. \& Thorburn, A. Autophagy and apoptosis- what's the connection? Trends Cell Biol. 21, 387-92 (2011).

48. Mariño, G., Nisosantano, M., Baehrecke, E. H. \& Kroemer, G. Self-consumption: the interplay of autophagy and apoptosis. Nat. Rev. Mol. Cell Biol. 15, 81-94 (2014).

49. Rashid, H. O., Yadav, R. K., Kim, H. R. \& Chae, H. J. ER stress: autophagy induction, inhibition and selection. Autophagy 11, 1956-1977 (2015)

50. Von, S. S., Nardin, A., Schrepfer, E. \& Ziviani, E. Mitochondrial dynamics and mitophagy in Parkinson's disease: a fly point of view. Neurobiol. Dis. 90, 58-67 (2015).

51. Ni, H. M. et al. Bid agonist regulates murine hepatocyte proliferation by controlling endoplasmic reticulum calcium homeostasis. Hepatology $\mathbf{5 2}$, 338-348 (2010)

52. $\mathrm{Ni}, \mathrm{H}$. M. et al. Bid regulates murine hepatocyte proliferation by controlling ER calcium homeostasis. Hepatology 52, 338-348 (2010).

53. Elmore, S. P., Qian, T., Grissom, S. F. \& Lemasters, J. J. The mitochondrial permeability transition initiates autophagy in rat hepatocytes. FASEB J. 15, 2286-2287 (2001).

54. Cohen, J. C., Horton, J. D. \& Hobbs, H. H. Human fatty liver disease: old questions and new insights. Science 332, 1519-1523 (2011)

55. Pugazhenthi, S. et al. Akt/protein kinase B up-regulates $\mathrm{BCl}-2$ expression through CAMP-response element-binding protein. J. Biol. Chem. 275, 10761-10766 (2000)

56. KHVousdenDPLane2007p53 in health and diseaseNat. Rev. Mol. Cell Biol.8275283Vousden, K. H. \& Lane, D. P. p53 in health and disease. Nat. Rev. Mol. Cell Biol. 8, (275-283 (2007).

57. Gori, M. et al. Protective effect of the Y220C mutant p53 during steatosis: Good news? J. Cell Physiol. 230, 1182-1192 (2015).

58. Panasiuk, A., Dzieciol, J. \& Panasiuk, B. et al. Expression ofp53, Bax and Bcl-2 proteins in hepatocytes in non-alcoholic fatty liver disease. World J. Gastroenterol. 12, 6198-6202 (2006).

59. Gahl, R. F., Dwivedi, P. \& Tjandra, N. Bcl-2 proteins bid and bax form a network to permeabilize the mitochondria at the onset of apoptosis. Cell Death Dis. 7, e2424 (2016).

60. Decuypere, J. P. et al. Ins(1,4,5)P3 receptor-mediated Ca2+ signaling and autophagy induction are interrelated. Autophagy 7, 1472-1489 (2011).

61. Lazarou, M. et al. PINK1 drives Parkin self-association and HECT-like E3 activity upstream of mitochondrial binding. J. Cell Biol. 200, 163-172 (2013).

62. Xie, F. et al. ASPP2 attenuates triglycerides to protect against hepatocyte injur by reducing autophagy in a cell and mouse model of non-alcoholic fatty liver disease. J. Cell. Mol. Med. 19, 155-164 (2015).

63. Zhang, $X$. et al. Functional proteomic analysis of nonalcoholic fatty liver disease in rat models: enoyl-coenzyme a hydratase down-regulation exacerbates hepatic steatosis. Hepatology 51, 1190-1199 (2010). 